\title{
La France et l'Europe : indépendance alimentaire dans un marché ouvert
}

\author{
Christian PÈES \\ Euralis Avenue Gaston Phoebus, \\ 64231 Lescar cedex \\ <christine.anglade@euralis.fr>
}

\author{
Abstract: How to defend European agriculture, against the dogmatism of the market and the lack of \\ ecological realism. The point of view of a farmer, chairman of the agro-food groupEuralis. \\ Key words: European agriculture, agrofood, food independance
}

Je suis agriculteur dans le sud-ouest de la France dans le cadre d'une exploitation associant culture du maïs et élevage de porc. Je suis aussi le président d'un grand groupe coopératif du Sud-Ouest, Euralis, qui est assez éclectique puisque nous réunissons les métiers de base de l'agriculture en passant par la collecte de céréales, etc., et des activités plus sophistiquées de transformation: nous sommes par exemple numéro un mondial du foie gras.

Je vous parlerai donc en homme de terrain, du point de vue de l'exploitant agricole et du point de vue du président d'une grosse entreprise en voie d'internationalisation.

Michel Griffon m'a bien simplifié la tâche en dressant le tableau du contexte mondial de l'agriculture auquel je souscris complètement. Nous sommes effectivement face à un formidable défi : nourrir dans l'avenir le plus correctement possible cette humanité future de 9 milliards d'habitants, et dès maintenant les 6,5 milliards d'aujourd'hui et cela tout en préservant de manière active l'environnement. Ces deux contraintes sont étroitement liées. Ce qui veut dire en pratique que les techniques qui ont été employées en particulier en France et en Europe jusqu'à présent ne seront plus réutilisables en l'état et qu'il nous faudra les modifier de façon considérable. C'est à ce point que nous rencontrons ambiguités et contradictions.

Michel Griffon a donné en exemple le labour une opération de désherbage, rappelons-le. Cette opération effectivement intervient pour beaucoup dans les bilans économiques et énergétiques d'une exploitation. Dans le contexte actuel, c'est sûr, il ne faut plus labourer. Le problème est que, dans le même temps, il nous est fortement conseillé de ne plus recourir aux produits phytosanitaires, aux désherbants. Et là, face à cette double contrainte, je l'avoue, je ne sais plus faire. Michel Griffon soutient avec raison qu'il ne faut plus labourer et simultanément d'autres critiquent avec la même conviction l'usage du Roundup ${ }^{\odot}$ ou d'autres désherbants. Telle est la situation qu'il nous faut apprendre à gérer et qui caractérise bien la problématique actuelle qu'il nous faut faire évoluer.

Les enjeux actuels appellent ainsi des sauts technologiques considérables. Ils impliquent tout autant une large prise de conscience sur le plan politique et économique. Or, à quoi assiste-t-on en France et en Europe actuellement?

Je prendrais deux exemples, celui de l'évolution de la PAC et celui des relations entre agriculture et environnement tel qu'il se dégage à I'issue du Grenelle de l'environnement. Vous le savez, l'année prochaine verra une évolution très sensible de la PAC, une évolution présentée comme intermédiaire mais qui sera vraisemblablement assez définitive et qui est peut-être déjà à I'heure actuelle complètement arrêtée. Mon inquiétude, compte tenu de ce qui a été dit précédemment du contexte mondial, vient du fait que l'Europe ne semble pas consciente de cette situation, $n$ 'inscrit pas cet enjeu stratégique au cœur de la PAC. Pourquoi ? Parce que les instances européennes en sont toujours à défendre l'idée d'un marché qui arbitrerait tout et en particulier le sort de l'agriculture. Que l'emportent les plus performants, le marché pourvoira au reste, y compris en Europe ! Ainsi, face aux défis qui s'annoncent, on fait comme si l'agriculture européenne n'était plus fondamentalement nécessaire sur la planète. Et des notions de dépendance ou d'autosuffisance alimentaires, il n'en est même plus question. Or, cette conception je le crois $s^{\prime} e s t$ construite dans une période où nous étions encore dans l'illusion de l'abondance. La réalité nous rattrape. On peut discuter sur les chiffres mais globalement je suis convaincu que nous sommes aujourd'hui face à un vrai problème de production de matières premières de base. Au niveau de la PAC, l'évolution en cours est porteuse de risques, certes pour les agriculteurs européens - ce qui n'est pas un problème majeur car nous ne sommes plus très nombreux, d'autres populations ont disparu - mais également pour les citoyens français, pour le consommateur français de demain. D'un côté, on met en avant un modèle alimentaire et de l'autre on le déstructure. Ce qu'il faut savoir c'est que si l'on abandonne la production des matières premières on abandonne aussi à la suite toute notre agro-industrie. Certains pensent (rêvent) qu'il est possible de maintenir les outils de transformation en Europe en important à bas coût la matière première, du Brésil ou d'Argentine par exemple. Je me souviens de discussions dans ce sens au sein de l'OMC. Seulement, les Brésiliens savent aussi faire de l'élevage et bâtir des abattoirs, des unités de découpe et de transformation sur les lieux de production. Et cette évolution est légitime, et tout à fait normale. Donc cette espèce de partage artificiel de la planète ne tient pas et en tout cas pas pour l'agriculture. Et c'est là que réside le danger pour les consommateurs européens. Ce qui se cache derrière tout cela, ce qui $m^{\prime}$ horripile, c'est que cela exprime un manque de vision, de colonne vertébrale, de stratégie. Imaginez le monde tel qu'il commence à se dessiner. L'Amérique du Sud dispose d'un gros potentiel. Les pays à l'est de l'Europe représentent certainement le gros potentiel de demain. J'en suis d'autant plus persuadé que notre groupe investi là-bas, mais imaginez ce que cela signifie. Je ne suis pas expert en politique étrangère, mais la Russie avec Poutine est déjà le premier exportateur de gaz et le deuxième de pétrole. On peut avoir de grandes idées sur la démocratie, l'organisation de la planète, il n'empêche que cette main-mise sur le robinet nous incline à faire quelques concessions. Je prends cet exemple parce demain, dans trente ans, la Russie sera sans aucun doute également un des grands greniers du monde, grenier à blé, à colza, à tournesol, voire à maïs, et l'arme alimentaire viendra s'ajouter à ces redoutables moyens de pression.

II nous faut donc une vision au niveau de l'Europe et nous ne pouvons nous contenter de devenir ce lieu idéal où on laisserait au marché le soin de tout arbitrer. Cela ne veut pas dire que je défends mordicus le système de subventions tel qu'il a fonctionné depuis trente ans. Ce n'est pas l'objet de notre discussion 
aujourd'hui. Je sais qu'il génère ici ou là beaucoup d'injustices. Non ce n'est pas cela que je défends. En tant qu'agriculteur, en tant que patron d'entreprise, ce que je veux avant tout, avant même les subsides, ce sont des règles de marché équitables. Et là ne soyons pas naiifs. Si je regarde ce qui se fait sur la planète, nulle part, et particulièrement chez les gens qui se réclament le plus du libéralisme, on ne laisse faire le marché.

Je pourrais multiplier les exemples, mais avant tout "cessons d'être naîfs". Nous avons besoin d'outils de régulation, nous avons besoin d'une règle du jeu, si possible planétaire, et si nous ne pouvons pas l'obtenir, maintenons au moins des garde-fous au niveau de I'Europe. Tout simplement pour préserver notre potentiel de production et de transformation. Si je traduis en emplois la production agricole c'est effectivement epsilon, $1 \%, 2 \%$ des emplois aujourd'hui mais si je replace en aval l'agroalimentaire et tous les services, on atteint $18 \%$ des emplois dans un pays comme la France. Et c'est cela qui est en jeu. Je ne m'arrêterai pas de le répéter: "Ayons une vision, battons-nous pour organiser cette vision et dotons-nous des outils nécessaires». Mais il ne me semble pas que l'on prenne cette direction. Ceci pour l'évolution de la PAC.

Le Grenelle de l'environnement: j'ai évoqué, avec le cas du labour, le type de contradictions auxquelles pouvait conduire ce genre de discussions. Le problème environnemental est réel bien évidemment. Mais je crains que les conclusions ne soient pas équilibrées, pas autant qu'elles devraient l'être. L'environnement est un des piliers du développement durable pour l'agriculture, mais ce n'est pas le seul. Or la question sociétale a été peu abordée, la dimension économique encore moins. Ainsi lorsqu'est proposé un objectif de $20 \%$ de surface bio en France dans 10 ans, alors qu'il n'y en que 2 ou $3 \%$ aujourd'hui, je crains que cela ne soit pas réaliste. On s'est fixé sur des statistiques arrêtées en 2004, à l'époque où étaient pris en compte les agriculteurs qui s'étaient tournés vers le bio en 1998-1999, avec l'opportunité du $\mathrm{CTE}^{1}$. Or, $90 \%$ de ces agriculteurs

\footnotetext{
${ }^{1}$ Les contrats territoriaux d'exploitation, pour une durée de 5 ans mis en place par la loi d'orientation agricole de 1999 (NDLR)
}

sont aujourd'hui revenus à des modes de production non-bio, tout simplement parce qu'ils ne «s'en sortent pas » en termes de revenus. Nous avons un groupe bio important chez Euralis, car il y a un potentiel, mais ne rêvons pas, pour devenir un secteur rentable les prix du bio devraient être doublés. Et là nous nous heurtons à un problème de pouvoir d'achat. Et pour ajouter à cette situation, comme en France on ne déteste pas être un peu intégriste en matière de cahier des charges du bio, ce sont nos camarades, italiens entre autres, plus pragmatiques, qui exporteront chez nous et prendront les marchés.

Sur un autre plan, réduire les produits phytosanitaires est un objectif réalisable à condition de pouvoir disposer de technologies de substitution. Les OGM sont une de ces solutions et constitueront dans les deux décennies à venir l'outil le plus puissant pour résoudre un certain nombre de problèmes. Je ne devrais pas dire "OGM », mais « biotechnologie », qui recouvre un champ beaucoup plus large dont les OGM ne sont qu'une partie. La génétique est au cœur de beaucoup de choses, de beaucoup d'enjeux pour demain. Demain c'est grâce à la génétique que nous disposerons de plantes consommant moins d'engrais. Aujourd'hui, pour acheter du P2K, il faut se rendre très loin au fond de la Sibérie, dans des circuits commerciaux dont je vous laisse imaginer la complexité. Or nous avons des outils de recherche, et déjà des exemples concrets d'outils permettant d'avancer. J'ai vu aux Etats-Unis des cultures de maïs à rendement constant, c'est-à-dire 100-110 quintaux par hectare et consommant quatre fois moins d'azote. Cela m'intéresse en tant qu'agriculteur car cela me coûtera moins cher, cela m'intéresse en tant que consommateur car cela me coûtera normalement aussi moins cher et cela m'intéresse en tant que citoyen car quatre fois moins d'azote sur le maïs signifie quatre fois moins de nitrates dans les eaux. On peut citer de nombreux exemples comme celui-ci.

Tout ce qu'a décrit Michel Griffon constitue notre réalité, ce défi nous devons le relever. Mais de grâce, relevons-le de façon pragmatique, regardons les problèmes, on ne les traitera pas tous c'est évident, on n'arrivera pas malheureusement à nourrir correctement les 9 milliards d'habitants de 2050, on n'arrive déjà pas à bien en nourrir 6 milliards. Mais j'espère que I'on progressera et on n'y arrivera qu'à la condition de garder les yeux ouverts et d'avoir la volonté de traiter les problèmes. Or aujourd'hui on n'aborde généralement pas ces problématiques avec la volonté de trouver une solution. Je crois qu'il est possible de préserver les choses, et que la place de l'agriculture européenne et française est vitale. $Y$ compris et surtout en cette période de changement climatique, qui sera loin d'être uniforme. Quoi qu'il en soit des conséquences extrêmement variées qui s'annoncent, l'Europe et la France en particulier devraient garder leur régime tempéré.

Nous sommes dans une zone tempérée, cela mérite qu'on y accorde de l'attention, qu'on la protège et c'est pour cela que j'affirme qu'il serait criminel de réduire le potentiel de production de l'Europe. Il y va de nos emplois mais aussi des productions que nous pouvons apporter au monde. Vous aurez compris que mon inquiétude aujourd'hui réside dans le fait de ne pas savoir vers quoi l'on va, de constater que nous sommes dans une approche de ces problèmes que je qualifierai d'hystérique. On décrit des calamités, on décrit des drames possibles, mais nous ne sommes pas raisonnables dans l'approche. Vous êtes de milieux très divers, d'origines très diverses, la seule chose que je voudrais, c'est que vous compreniez cela. Ne nous laissons pas leurrer par tous ces discours de marchands d'apocalypse, de peur, etc. Nous avons un chantier devant nous, nous devons nous atteler à la tâche, y mettre de I'huile de coude, favoriser l'innovation, car elle est au cœur de la résolution des problèmes, avec toutes les précautions qui s'imposent évidemment. Aujourd'hui l'agriculteur et le président d'une entreprise agroalimentaire que je suis, vous disent que l'on souffre et que nous avons peur quant à notre pérennité par rapport aux évolutions que l'on pressent alors que fondamentalement, comme l'a décrit Michel Griffon, la situation est excellente pour un agriculteur ou une entreprise agroalimentaire. $C^{\prime}$ est ce paradoxe que nous sommes en train de vivre. 